\title{
State-of-health monitoring of lithium-ion battery modules and packs via incremental capacity peak tracking
}

\author{
Caihao Weng ${ }^{\mathrm{a}, *}$, Xuning Feng ${ }^{\mathrm{ab},{ }^{, * *},}$ Jing Sun ${ }^{\mathrm{a}}$, Huei Peng ${ }^{\mathrm{b}, \mathrm{c}}$ \\ Corresponding Author:*chsweng@umich.edu,**fxn07@mails.tsinghua.edu.cn \\ a Department of Naval Architecture and Marine Engineering, University of Michigan, Ann Arbor, \\ Michigan, 48109, USA \\ b State Key Laboratory of Automotive Safety and Energy, Tsinghua University, Beijing, 100084, China \\ c Department of Mechanical Engineering, University of Michigan, Ann Arbor, Michigan, 48109, USA
}

\begin{abstract}
Incremental capacity analysis (ICA) is a widely used technique for lithium-ion battery state-of-health $(\mathrm{SOH})$ evaluation. The effectiveness and robustness of ICA for single cell diagnostics have been reported in many published work. In this study, we extend the ICA based SOH monitoring approach from single cells to battery modules, which consist of battery cells with various aging conditions. In order to achieve on-board implementation, an IC peak tracking approach based on the ICA principles is proposed. Analytical, numerical and experimental results are presented to demonstrate the utility of the IC peak tracking framework on multi-cell battery $\mathrm{SOH}$ monitoring and the effects of cell non-uniformity on the proposed method. Results show that the methods developed for single cell capacity estimation can also be used for a module or pack that has parallel-connected cells.
\end{abstract}

\section{Key words}

Lithium-ion batteries; State-of-health; Incremental capacity analysis; Battery pack 


\section{Introduction}

Battery state of health ( $\mathrm{SOH}$ ) monitoring has become an important research area [1-5]. SOH is a measure that reflects the current status of a battery, including the capacity and the capability of power output, in comparison with its fresh status $[6,7]$. Take electric vehicle as an example, the current capacity directly links with the driving range of the electric vehicle, whereas the capability of power output determines the dynamic property of the electric vehicle. The capability of power output is determined by both the capacity $(Q)$ and the resistance $(R)$ of the battery, therefore the key task for the SOH monitoring is to estimate the $Q$ and $R$ online [8].

The battery SOH continuously deteriorates due to irreversible physical and chemical changes in its life cycle. The aging process typically involves multiple mechanisms that affect both capacity and resistance of the battery [9], leading to the reduction of the battery's energy and power density. In the case of lithium ion cells, the performance degradation could be caused by many phenomena such as the growth of solid electrolyte interface (SEI) layers, loss of active materials, electrolyte decomposition, and electrode structural disordering [10-14]. Most of those mechanisms are coupled during the aging process and cannot be isolated and studied independently, thereby making the investigation of battery aging mechanism challenging [10]. On the other hand, good understanding of battery aging mechanisms and accurate detection of $\mathrm{SOH}$ are needed for design of battery management strategies to maintain performance, safety and long life cycle $[15,16]$.

Whereas the resistance could be assessed on-line using identification algorithms such as least squares methods [17-19], the detection of capacity fading requires columbic counting of a full charge/discharge cycle[20,21], which is hard to be satisfied in practical on-line conditions. In 
recent years, incremental capacity analysis (ICA) has become a popular approach for evaluating battery capacity loss in both laboratory and on-board applications [7, 20, 22-24].

ICA, which was first developed for studying the properties of the intercalation based battery cells [25-27], transforms voltage plateaus on the charging/discharging curve into peaks on the incremental capacity (IC) curve [28]. ICA is applicable to various battery types and was shown to be an effective tool for analyzing the capacity loss of single lithium ion cells. In practice, however, battery cells are always connected in various series-parallel configurations to form battery modules and packs, especially for high power applications such as in hybrid electric vehicles. The SOH monitoring of multi-cell battery modules is therefore crucial in practical operations.

The main difference between a single cell and a module that has parallel connected cells, when applying the ICA, is the existence of uneven current distribution due to the cell non-uniformity. Consequently, the current going through each cell in the module could be time varying even when the module is charged at a constant current [29]. Since ICA typically requires constant charging/discharging data, the uneven distribution could affect the applicability of ICA to a battery module. For parallel-connected cells, given that only the total current, rather than the individual cell currents, is measured online, performing ICA for individual cells to determine the total module capacity is not an option [30]. Therefore monitoring $\mathrm{SOH}$ for batteries connected in a module requires tools and algorithms beyond what have been learned for single cells.

In this paper, an IC peak tracking framework based on the ICA principles is proposed to monitor SOH on-board for battery modules and packs. The applicability of the framework is investigated using both simulations and experimental results. The investigation is based on lithium iron phosphate $\left(\mathrm{LiFePO}_{4}\right)$ cells with various aging conditions. Cells with different aging history 
are combined to form battery modules with different capacity to test and validate the proposed methodology.

The remainder of this paper is organized as follows. In Sections 2 and 3, the ICA based SOH monitoring framework designed for single cells is discussed and the applicability of the framework to battery module is analyzed. In Section 4, the application of ICA for battery module $\mathrm{SOH}$ monitoring is verified through simulation using a module model incorporating cell aging mechanism. Then the experimental design and setup for battery module tests are presented in Section 5. The experimental ICA results are discussed and analyzed in Section 6. Finally, the conclusions are given in Section 7.

\section{ICA for Single Cell SOH Monitoring}

The concept of ICA originates from the study of the intercalation process based batteries [25-27, 31]. Using graphite based lithium ion cell as an example, during the charging (or discharging) process, when the lithium ions are being intercalated into (or de-intercalated from) the carbon electrode, the graphene sheets together with the solid phase lithium ions are arranged periodically to form different stage structures [32]. Those stages are associated with different energy levels in the negative electrode, and reflected as multiple voltage plateaus on the battery OCV curve. The ICA technique can be used to characterize the electrochemical properties related to the intercalation process by computing the IC curve as,

$$
I C=\frac{d Q}{d O C V}
$$

and transforming the voltage plateaus into clearly identifiable peaks, where $Q$ represents the charged capacity. In practice, the OCV data are often substituted with voltage data (V) collected with constant current (for instance, 1/25 $\mathrm{C}$ rate) under slow charging/discharging, sometimes 
referred as close to equilibrium OCV data [22]. The $\mathrm{d} Q / \mathrm{d} V$ based ICA curve could accurately reflect the characteristics of the underlying battery chemistry, given that the results are properly computed, due to the following equivalence,

$$
\frac{d Q}{d V}=\frac{d Q}{d(O C V+I R)} \approx \frac{d Q}{d O C V}=I C
$$

where $V, I, R$ are the battery voltage, current and internal resistance, and $\mathrm{d} I R \ll \mathrm{d} O C V$ when $\mathrm{d} I$ is small.

ICA has the advantage to detect a gradual change in cell behavior during aging and degradation [22, 33, 34]. It is useful particularly for battery SOH monitoring as the extracted peak values and their change pattern are closely related to the battery capacity fading. Fig. 1 shows the IC curves for a series of degradation test from [7]. It can be seen that the second IC peak drops monotonically as capacity degrades. The correlation between the IC peak intensities and battery faded capacity can be used to formulate a battery capacity estimation model [20, 34]. Through the identification of IC peaks using battery charging data, one can estimate the capacity and evaluate the battery health condition for single cells. One of the major difficulties in implementing ICA is the computation of the IC curve directly from the test data on-board. This issue has been addressed by different approaches including the probability density function method in [23], the support vector regression method in [7] as well as using the high precision charger [35, 36].

In this paper, we will extend the methodology developed with single cells to battery modules for cells connected in parallel.

\section{Multi-Cell SOH Monitoring with Cell Non-Uniformity}

\subsection{Backgrounds}


For single battery cells, the IC peak intensities and areas under the peaks are quantitatively correlated to cell capacity and therefore could be used to identify capacity degradation [7]. The ICA based capacity estimation approach (by tracking the changes of the IC peak values) could be extended from cells to packs if the same correlation between capacity and IC peaks can be established for a module or pack.

Most popular battery pack configurations connect cells in parallel first to form small modules, and then connect the modules in series to form the pack [37,38]. For instance, as shown in Fig. 2, the Chevrolet Volt's pack is made up of 96 modules with each module having 3 cells connected in parallel, and Nissan Leaf has modules that contain 2 in-series cell pairs connected in parallel [37]. The parallel connected cells can self-balance their SOC throughout the operations. The configuration also improves the overall robustness of battery pack. However, in such design, the currents going through individual cells are usually not monitored and only the terminal data can be used for battery state monitoring [29]. As the individual cell measurements are no longer available, the IC peak tracking based battery capacity estimation framework has to be performed using the terminal data. However, cell-to-cell variation is inevitable in battery productions [39-43]. The variation might grow larger, as the aging rate of each single cell could be different during operations. The non-uniformity could lead to uneven current distribution among the parallel-connected cells, thereby making $\mathrm{SOH}$ monitoring more challenging compared to the single cell case. In this paper, battery module refers to several parallel-connected cells and our investigation focuses on the applicability of IC peak tracking to the module with only terminal measurements, and how cell non-uniformity in the module could affect the application. It should also be noted that inside a battery cell, similar characteristics as discussed in Sec. 3.2 may exist 
due to unequal degradation from layer to layer, and this may be of interesting for further investigation.

From our previous work, an inventory of more than 30 test cells at different aging stage is established [7]. The batteries available for this study are $\mathrm{LiFePO}_{4}$ cells from $\mathrm{A} 123$ with the specifications listed in Table 1. The battery cells have different degrees of degradation after hundreds of cycles of aging tests, as described in [7]. The distribution of the cell capacity and resistance of the inventory is plotted in Fig. 3. One can see that the cells have capacities varying from $1.1 \mathrm{Ah}$ to $0.8 \mathrm{Ah}$, and internal resistance varying from $0.12 \Omega$ to $0.17 \Omega$. Battery modules with distinctive cell combinations are built for the study.

\subsection{Influence of Non-uniformity in Applying IC Peak Tracking to Battery Modules}

The applicability of ICA based methods to battery modules is first explored using analytical analysis. To simplify the analysis, let's consider the simple model,

$$
\begin{aligned}
& V_{k}=O C V_{k}+R_{0} I_{k} \\
& Q_{k+1}=Q_{k}+I_{k} \Delta t
\end{aligned}
$$

where $V_{k}, I_{k}, Q_{k}$ are the voltage, current, and the charged capacity at sample time $k$ respectively. $R_{0}$ is the battery internal resistance and $\Delta t$ is the time step. Then for an individual battery cell, the IC curve can be computed as the following when constant charging current is applied,

$$
I C_{k}=\frac{\Delta Q_{k}}{\Delta O C V_{k}}=\frac{\Delta Q_{k}}{\Delta O C V_{k}+R_{0} \Delta I_{k}}=\frac{\Delta Q_{k}}{\Delta V_{k}}
$$

where $\Delta I_{k}=0$ when $I_{k}$ is a constant current. The IC curve calculated using terminal measurement data $\left(\Delta Q_{k} / \Delta V_{k}\right)$ could well represent the battery characteristics reflected by the OCV-SOC function.

On the other hand, for a battery module with 2 parallel-connected cells, we have, 


$$
\begin{aligned}
& \frac{\Delta Q_{k}}{\Delta V_{k}}=\frac{\Delta Q_{1 k}+\Delta Q_{2 k}}{\Delta V_{k}}=\frac{\Delta Q_{1 k}}{\Delta V_{1 k}}+\frac{\Delta Q_{2 k}}{\Delta V_{2 k}} \\
& =\frac{\Delta Q_{1 k}}{\Delta O C V_{1 k}+R_{0,1} \Delta I_{1 k}}+\frac{\Delta Q_{2 k}}{\Delta O C V_{2 k}+R_{0,2} \Delta I_{2 k}} \\
& <\frac{\Delta Q_{1 k}}{\Delta O C V_{1 k}}+\frac{\Delta Q_{2 k}}{\Delta O C V_{2 k}}
\end{aligned}
$$

where 1 and 2 in the subscript indicate the cell \#1 and \#2 in the battery module respectively. $I_{1 k}$ and $I_{2 k}$ would vary with time even when a constant current is applied to charge the module, due to the uneven current distribution caused by the cell-to-cell variation. In this case, $\Delta I_{1 k}$ and $\Delta I_{2 k}$ are no longer zero in the battery module. The $\Delta Q_{k} / \Delta V_{k}$ calculation may not capture the aging signature embedded in the battery cells' OCV value. Moreover, the IC peaks for the two cells would appear at different time during the charging process. According to the inequality in Eqn. (5), the uneven current leads to broadening of the IC curve. This could further distort the correlation between the module IC peak value identified from the terminal measurements of terminal and the variables of the module, and thereby affecting the estimation of the actual battery module capacity.

The applicability of ICA based methods for module SOH monitoring, therefore, critically relies on understanding the effects of the cell uniformity. In order to gain insights on how the variations in cell current affect the $\mathrm{SOH}$ monitoring framework, simulation analyses are performed using a battery module model in the following section.

\section{IC Peak Tracking for Battery Module SOH Characterization: Simulation Analysis}

The objective of the simulation analysis is to explore how the IC curve of a battery module changes with different module capacity and cell non-uniformity. The goal is pursued with a battery model that emulates the behavior of a module with cells of different characterizations.

\subsection{Battery Module Model}


The battery module model is constructed based on the approach proposed in [44] as shown in Fig. 4 for a 3 -cell ${ }^{1}$ module system. The single cell behavior is simulated using an equivalent circuit model (ECM). A first-order ECM with one RC circuit can be described as follows [45,46],

${ }^{I}$ The 3-cell module system is built to emulate the module design of the Chevy Volt.

$$
V_{k}=O C V\left(z_{k}\right)-V_{R C, k}+R_{0} I_{k}
$$

Where

$$
\begin{aligned}
& z_{k+1}=z_{k}+\left(\frac{\eta_{i} \Delta t}{Q}\right) I_{k} \\
& V_{R C, k+1}=V_{R C, k} \cdot \exp \left(\frac{-\Delta t}{R_{1} C}\right)-R_{1} I_{k} \cdot\left[1-\exp \left(\frac{-\Delta t}{R_{1} C}\right)\right]
\end{aligned}
$$

$z_{k}$ is the state of charge (SOC), $\eta_{i}$ is the Columbic efficiency, $\Delta t$ is the time step, $Q$ is the battery capacity, $I_{k}$ is the current, $V_{k}$ is the terminal voltage, $V_{R C, k}$ is the voltage of the RC circuit, $R_{0}, R_{1}$ and $C$ are battery internal resistance and capacitance.

The OCV function that incorporates the battery aging effects is simulated using the mechanistic battery aging model proposed in [47]. The battery model considers the aging mechanism of both the positive and negative electrodes, and reflects the qualitative relationship between the equilibrium potentials and battery aging status [34]. The analytic models for the equilibrium potentials of $\mathrm{LiFePO}_{4}$ batteries can be found in the literature $[48,49]$. The overall equilibrium potential of the battery cell is the difference between the positive electrode and the negative electrode, $V_{\text {total }}=V_{\mathrm{PE}}-V_{\mathrm{NE}}$ (see Fig. 5).

As reported in $[28,50-53]$, the capacity fading of $\mathrm{LiFePO}_{4}$ cells is dominated by the loss of lithium inventory. The degradation of the carbon negative electrode has little effect on the cell capacity for LFP cells as reported in $[34,50]$. The loss of lithium inventory is directly reflected in the reduction of the second IC peak, as shown in Fig. 1. 
Therefore, loss of lithium inventory is simulated as the main capacity degradation mechanism in our aging analysis. And the magnitude of the second IC peak is regarded as an effective indicator to evaluate the capacity of the battery.

For a 3-cell module, following the Kirchhoff's laws, the voltages and currents of the parallel connected circuits satisfy:

$$
\begin{aligned}
& V_{s, 1}=V_{s, 2}=V_{s, 3} \\
& \alpha_{s, 1}+\alpha_{s, 2}+\alpha_{s, 3}=I_{\text {total }}
\end{aligned}
$$

where $V_{s, 1}, V_{s, 2}, V_{s, 3}$ are the string terminal voltage measurements, and $\alpha_{s, 1}, \alpha_{s, 2}, \alpha_{s, 3}$ are string currents. $I_{\text {total }}$ is the total module current.

The voltage response and current distribution are thereby computed as a solution of a set of linear equations [44]. The system of linear equations can be described by,

$$
\square\left(\begin{array}{l}
\alpha_{s, 1} \\
\alpha_{s, 2} \\
\alpha_{s, 3}
\end{array}\right)=\left(\begin{array}{ccc}
R_{0,1} & -R_{0,1} & 0 \\
0 & R_{0,2} & -R_{0,3} \\
1 & 1 & 1
\end{array}\right)^{-1}\left(\begin{array}{c}
\Pi_{s, 2}-\Pi_{s, 1} \\
\Pi_{s, 3}-\Pi_{s, 2} \\
I_{\text {total }}
\end{array}\right)
$$

where

$$
\Pi_{s, i}=O C V_{s, i}-V_{R C[s, i]}
$$

where $V_{R C}$ is defined in Eqn. (6).

\subsection{Tracking IC Peak Values of Battery Modules with Parallel Connected Cells}

Battery modules with different total capacity and non-uniformity (in both capacity and resistance) are simulated using the model described in the previous subsection. The results of five battery modules with different cell combinations are presented here. The three cells in each module are assumed to be connected in parallel as shown in Fig. 4. The cell capacities and resistance used in the module simulations are listed in Table 2. The internal resistance value 
represents the sum of $R_{0}$ and $R_{1}$ for each cell. The range of the capacity and resistance variation is selected based on the parameter values of the test cells (see Fig. 3). The correlation between the IC peak values (the magnitude of the IC peak) and the capacity of the simulated battery modules are compared as shown in Fig. 6 and 7.

The ICA results of battery modules \#S1, \#S2 and \#S3 are shown in Fig. 6. The three simulated modules have variation in both cell and total capacities but not in resistance. As marked by the arrow in Fig. 6, the value of second IC peak reduces as the module capacity degrades, which is consistent with the single cell results. The influence of non-uniformity in cell capacity is rather small and inconsequential. Therefore, without resistance variation, the ICA based capacity estimation is directly applicable to battery modules.

The sensitivity of the module IC peak values to non-uniformity in resistance is also investigated. Although the resistance change does not affect the ICA results for single cells, the difference between cells could be large and might affect the module ICA results because they could affect the current distribution. For the case studies, variations of $0.01 \Omega$ (Module \#S4) and $0.02 \Omega$ (Module \#S5) are simulated and the results are shown in Fig. 7. It is observed that, when the variation of internal resistance increases, the intensities of the IC peaks tend to decrease.

To better understand the quantitative relationship between the cell variation and the deviations in the IC peak values, extensive simulations are performed with battery modules that have parallel-connected cells. For the convenience of visualization, 2 instead of 3 cells are included in each simulated module. That is in a simulated module, there are Cell 1 and Cell 2 with variations in their capacities and resistances. A module that has two identical cells with identical capacity and resistance is called a reference module. The other simulated modules simulated have 
the same total capacity as the reference module, but different individual capacity for Cell 1 and Cell 2. Note that though the IC peak may decrease due to cell-to-cell variations, the IC curve may broaden to maintain a fixed area, given the same total capacity of the simulated modules.

The simulation is intended to capture the relationship between the accuracy of ICA with respect to cell capacity and resistance variation in a module. Consider the variation in the IC peak value using:

$$
\Delta I C=\frac{I C_{\mathrm{ref}}-I C_{\mathrm{var}}}{I C_{\mathrm{ref}}}
$$

where $I C_{\text {ref }}$ and $I C_{\text {var }}$ are the IC peak values of the reference module and the modules with non-uniform cells respectively.

The results are shown in Fig. 8, where $Q_{\text {var }}$ and $R_{\text {var }}$ denote the variations for the capacities and resistance of the cells in the modules, as defined in Eqn. (10) and (11), where $Q_{i}$ and $R_{i}$ are capacity and resistance of Cell $i(i=1,2)$, respectively. For the reference module, both $Q_{\mathrm{var}}$ and $R_{\mathrm{var}}$ equal to 1

$$
\begin{gathered}
Q_{\text {var }}=\frac{Q_{1}}{Q_{2}} \\
R_{\text {var }}=\frac{R_{1}}{R_{2}}
\end{gathered}
$$

The color code corresponds to the value of $\Delta I C$ as specified by the right-side bar in Fig. 8 . The $x$ and $y$ axes represent the variations in resistance $\left(R_{\mathrm{var}}\right.$ up to $\left.\pm 30 \%\right)$ and capacity ( $Q_{\mathrm{var}}$ up to $\pm 10 \%$ ) between the two cells in the module, respectively. The contour map (Fig. 8) shows that the deviation in the IC peak value could be up to $25 \%$ in the worst case. However, most of the changes in the battery module follow the pattern that increase in resistance is accompanied by the decrease in capacity. Therefore most of real-world non-uniform distributions are expected to fall 
within the neighborhood of the diagonal stripe ( $y=-x$ from the upper-left to the lower-right), and unlikely to fall into the other two corners (upper-right and lower-left) of Fig. 8, where the value of $\Delta I C$ is high. This is confirmed by experimental results. By plotting our battery data statistics onto the map, one can see that the range of deviation could be reduced to $10 \%$ (equivalent to $2 \%$ error in capacity estimation as shown later in Fig. 14).

The simulation results imply that the ICA based $\mathrm{SOH}$ monitoring framework developed for single cells can also be used for battery modules. The design of the validation test is presented in the next section.

\section{Experimental Setup}

The test data used for this study are acquired through the battery test bench set up in [54], which includes an Arbin BT2000 tester, a thermal chamber for environment control, a computer for user-machine interface and data storage, a switch board for cable connection, and battery cells. The data acquisition system has a sampling frequency of $10 \mathrm{~Hz}$.

Battery modules in experiment are formed by selecting aged cells from our test cell inventory and connecting them in parallel (see Fig. 9). In this work, total of about 30 modules were tested with 3 cells connected in parallel. The characteristics of the 3-cell modules are shown in Fig. 10, where the variation of individual cell capacity in a module is quantified using the standard deviation. One can see that the modules cover a wide range of capacity variations (2.5Ah to 3.2Ah) and have different variations. Some modules are made up of cells with similar aging status, while others have capacity variation up to $0.14 \mathrm{Ah}$ (more than $10 \%$ of the nominal capacity).

All tests are conducted at $24^{\circ} \mathrm{C}$. ICA is performed on the data to obtain the relationship between the IC curve and the battery degradation. It should be noted that $24^{\circ} \mathrm{C}$ is the temperature 
set for the thermal chamber, not the temperature of the individual cells in the 3-cell module. The temperature of the individual cells in the 3-cell module may vary with the cell resistances. The temperature variance and its influence on the cell/module performance require further investigation and will not be included in this manuscript.

\section{Results and Discussions}

The IC curves of the battery modules cannot be computed directly from numerical derivative or parametric curve fitting due to the measurement noise. Instead, the IC curves are computed using partial charging data with specialized processing algorithm such as the support vector regression (SVR) algorithm [7,55].

Take each charging data set with current and voltage measurements, let $q, v$ be the input and output of the SVR model ( $q$ represents the battery charged capacity, $v$ is the measured voltage). The SVR model for the $V$ - $Q$ curve can be expressed as,

$$
v=\sum_{i=1}^{N} \beta_{i} \cdot \mathrm{K}\left(q_{i}, q\right)+\mu
$$

where $N$ is the number of data points in the data set, $\beta_{i}$ and $\mu$ are the model parameters, whose values are determined based on the data set, and $\mathrm{K}\left(q_{i}, q\right)$ is the selected kernel. In this study, the Gaussian radial basis function (rbf) kernel is used and is expressed as

$$
\mathrm{K}\left(q, q^{\prime}\right)=\exp \left(\frac{-\left\|q-q^{\prime}\right\|^{2}}{2 \sigma^{2}}\right)
$$

where $\sigma$ is the adjustable parameter for the kernel function.

By solving a linear programming based optimization problem, the parameters in model (12) can be determined and the model can be reduced to

$$
f(q)=\sum_{i=1}^{N_{s v}} \beta_{i} \mathrm{~K}\left(s v_{i}, q\right)+\mu
$$


where $s v$ is the support vector (SV) and $N_{s v}$ is the total number of SVs $\left(N_{s v} \ll N\right)$. Then, the IC curve can be computed from the fitted $V$ - $Q$ curve as follows:

$$
I C(v)=\frac{d q(v)}{d v}=\frac{1}{f^{\prime}(q(v))}=\frac{1}{\sum_{i=1}^{N_{s v}} \beta_{i} \mathrm{~K}^{\prime}\left(s v_{i}, q(v)\right)}
$$

As an example, Fig. 11 shows the IC curve of a 3-cell module identified through the SVR algorithm. More detailed discussion on the SVR approach for IC curve identification can be found in [55].

Fig. 12 shows the ICA results of three 3-cell battery modules. The specifications of those modules with 3 aged cells are listed in Table 3. As expected, the IC curves extracted from the test data show a clear aging trend. The second IC peak (circled in Fig. 12) values decreases as the module capacity reduces.

\subsection{Results of Module IC Peak Tracking}

As in the single cell case [7], we may record the value of the second IC peak and use that as a signature of battery capacity fading. In order to see the robustness of the ICA based capacity estimation method, two battery modules with same total capacity but different individual cell capacities are compared in Fig. 13. The characteristics of the two modules are listed in Table 3. The collective capacity is the total module capacity measured through Coulomb counting. One might notice that the collective module capacity does not equal to the algebraic sum of the individual capacities. This can be caused by the uneven current distribution during charging/discharging that could affect the usable capacity of the module. For the module capacity estimation, we use the value of the collective capacity.

As shown in Fig. 13, although the voltages corresponding to the IC peaks are different due to the difference in resistance, the second IC peak values of the two packs are almost identical. 
Therefore the second IC peak value seems to be a robust aging signature for $\mathrm{LiFePO}_{4}$ battery modules, as demonstrated by simulations and experimental results.

\subsection{Battery Capacity Estimation Model}

To quantify the correlation between capacity and the corresponding IC peak values for $\mathrm{LiFePO}_{4}$ cells, a fitted curve (dashed line in Fig. 14) is obtained using only the single cell test data. The quantitative correlation provides us a tool to estimate the battery capacity based on the IC peak values identified from the test data. To validate this estimation method with battery module data, normalization is performed. Both capacity and IC peak values obtained from the data are divided by the nominal capacity values (e.g., 3-cell module data are divided by 3.3Ah, the total capacity of three fresh normal cells). After normalization, we could plot the IC peak tracking results of single cells and modules on the same figure, as shown in Fig. 14. The maximum deviation between fitted correlation and the battery module data is about $2 \%$. The accuracy of the battery capacity estimation is shown in Table 4 . One can see that, although the estimation model is built based on the single cell data, the estimation error does not increase when the method is applied to 3-cell data. This result implies that, the correlation identified using single cell data is generalizable to battery modules with various numbers of cells, and could be used as a battery degradation model for capacity estimation. However, it should be noted that the technique in this paper focuses more on estimating the module capacity for health monitoring, it does not provide information on which cell is fading in case of module failure.

\section{Conclusions}

This paper reports the extension of IC peak tracking based SOH monitoring framework from single cells to multi-cell battery modules. The applicability of the framework to battery module is 
first investigated through model simulation and then validated using experimental data. A test battery inventory that consists of more than $30 \mathrm{LiFePO}_{4}$ cells with various aging conditions is established, and the test cells are combined into battery modules for the study. The results show that, the total capacity loss of a battery module can also be correlated with the IC curve peaks, and the correlation is the same as the single cell case. The findings suggest that the SOH monitoring framework based on IC peak tracking can be applied to battery modules and packs with different series-parallel configurations. Although the quantitative results presented in this study might only be true for $\mathrm{LiFePO}_{4}$ cells, the design of the framework is generalizable for cells with other chemistries.

\section{Acknowledgment}

This work is supported by the US Department of Energy under Award Number DE-PI0000012.

\section{References}

[1] Liaw BY, Dubarry M, in: G. Pistoia (Ed.), Electric and Hybrid Vehicles: Power Sources, Models, Sustainability, Infrastructure and the Market, Elsevier, 2010.

[2] Offer G J, Howey D, Contestabile M, et al. Comparative analysis of battery electric, hydrogen fuel cell and hybrid vehicles in a future sustainable road transport system. Energy Policy, 2010, 38(1): 24-29.

[3] Bai G, Wang P, Hu C, Pecht M. A generic model-free approach for lithium-ion battery health management. Applied Energy, 2014, 135: 247-260.

[4] Waag W, Fleischer C, Sauer D U. Critical review of the methods for monitoring of lithium-ion batteries in electric and hybrid vehicles. Journal of Power Sources, 2014, 258: 321-339.

[5] Ng SSY, Xing Y, Tsui KL. A naïve Bayes model for robust remaining useful life prediction of lithium-ion battery. Applied Energy, 2014, 118: 114-123.

[6] Pop V. Battery management systems: Accurate state-of-charge indication for battery-powered 
applications. Springer Science \& Business Media, 2008.

[7] Weng C, Cui Y, Sun J, et al. On-board state of health monitoring of lithium-ion batteries using incremental capacity analysis with support vector regression. Journal of Power Sources, 2013, 235: $36-44$.

[8] Ouyang M, Feng X, Han X, et al. A dynamic capacity degradation model and its applications considering varying load for a large format Li-ion battery. Applied Energy, 2016, 165: 48-59.

[9] Hu C, Youn BD, Chung J. A multiscale framework with extended Kalman filter for lithium-ion battery SOC and battery estimation. Applied Energy, 2012, 92: 694-704.

[10] Vetter J, Novák P, Wagner M R, et al. Ageing mechanisms in lithium-ion batteries. Journal of power sources, 2005, 147(1): 269-281.

[11] Moura S J. Techniques for battery health conscious power management via electrochemical modeling and optimal control. Pennsylvania State University, 2011.

[12] Feng X, Sun J, Ouyang M, et al. Characterization of large format lithium ion battery exposed to extremely high temperature. Journal Power Sources, 2014, 272: 457-467.

[13] Wu B, Yufit V, Merla Y, et al. Differential thermal voltammetry for tracking of degradation in lithium-ion batteries. Journal of Power Sources, 2015, 273: 495-501.

[14] Omar N, Monem MA, Firouz Y, et al. Lithium iron phosphate based battery - Assessment of the aging parameters and development of cycle life model. Applied Energy, 2014, 113: $1575-1585$.

[15] Offer G J, Yufit V, Howey D A, et al. Module design and fault diagnosis in electric vehicle batteries. Journal of Power Sources, 2012, 206: 383-392.

[16] Farmann A, Waag W, Marongiu A, et al. Critical review of on-board capacity estimation techniques for lithium-ion batteries in electric and hybrid electric vehicles. Journal of Power Sources, 2015, 281: 114-130.

[17] Kim J, Cho B H. State-of-charge estimation and state-of-health prediction of a Li-ion degraded battery based on an EKF combined with a per-unit system. Vehicular Technology, IEEE Transactions on, 2011, 60(9): 4249-4260.

[18] Remmlinger J, Buchholz M, Meiler M, et al. State-of-health monitoring of lithium-ion batteries in electric vehicles by on-board internal resistance estimation. Journal of Power Sources, 2011, 196(12): 5357-5363.

[19] Hu X, Sun F, Zou Y, Peng H, in: Proc. 2011 Am. Control Conf., San Francisco, CA.

[20] Groot J. State-of-Health Estimation of Li-ion Batteries: Ageing Models. Master Thesis, 
Chalmers University of Technology, 2012.

[21] Roscher M A, Assfalg J, Bohlen O S. Detection of utilizable capacity deterioration in battery systems. Vehicular Technology, IEEE Transactions on, 2011, 60(1): 98-103.

[22] Dubarry M, Svoboda V, Hwu R, et al. Incremental capacity analysis and close-to-equilibrium OCV measurements to quantify capacity fade in commercial rechargeable lithium batteries. Electrochemical and solid-state letters, 2006, 9(10): A454-A457.

[23] Feng X, Li J, Ouyang M, et al. Using probability density function to evaluate the state of health of lithium-ion batteries. Journal of Power Sources, 2013, 232: 209-218.

[24] Weng C, Sun J, Peng H. A unified open-circuit-voltage model of lithium-ion batteries for state-of-charge estimation and state-of-health monitoring. Journal of Power Sources, 2014, 258: 228-237.

[25] Thompson A H. Electrochemical potential spectroscopy: a new electrochemical measurement. Journal of The Electrochemical Society, 1979, 126(4): 608-616.

[26] Yazami R, Touzain P. A reversible graphite-lithium negative electrode for electrochemical generators. Journal of Power Sources, 1983, 9(3): 365-371.

[27] Dahn J R. Phase diagram of $\mathrm{Li}_{x} \mathrm{C}_{6}$. Physical Review B, 1991, 44(17): 9170.

[28] Dubarry M, Liaw B Y. Identify capacity fading mechanism in a commercial $\mathrm{LiFePO}_{4}$ cell. Journal of Power Sources, 2009, 194(1): 541-549.

[29] Gogoana R, Pinson M B, Bazant M Z, et al. Internal resistance matching for parallel-connected lithium-ion cells and impacts on battery pack cycle life. Journal of Power Sources, 2014, 252: 8-13.

[30] Gong X, Xiong R, Mi C C. Study of the characteristics of battery packs in electric vehicles with parallel-connected lithium-ion battery cells. Industry Applications, IEEE Transactions on, 2015, 51(2): 1872-1879.

[31] Barker J, Saidi M Y, Koksbang R. Differential capacity as a spectroscopic probe for the investigation of alkali metal insertion reactions. Electrochimica Acta, 1996, 41(16): 2639-2646.

[32] Schalkwijk W V, Scrosati B. Advances in lithium-ion batteries, Springer Science \& Business Media, 1st edition, 2002.

[33] Han X, Ouyang M, Lu L, et al. A comparative study of commercial lithium ion battery cycle life in electrical vehicle: Aging mechanism identification. Journal of Power Sources, 2014, 251: 38-54.

[34] Dubarry M, Truchot C, Liaw B Y. Synthesize battery degradation modes via a diagnostic and prognostic model. Journal of power sources, 2012, 219: 204-216.

[35] Smith A. A High Precision Study of Li-Ion Batteries, Ph.D. thesis, Dalhousie University, 2012 .

[36] Smith A J, Dahn J R. Delta differential capacity analysis. Journal of The Electrochemical Society, 2012, 159(3): A290-A293.

[37] Arcus C, A tale of 3 battery packs, 2016. 
http://cleantechnica.com/2016/01/06/a-tale-of-3-battery-packs/ ,accessed: 2016-05-06.

[38] Feng X, He X, Ouyang M, et al. Thermal runaway propagation model for designing a safer battery pack with $25 \mathrm{Ah} \mathrm{LiNi}_{x} \mathrm{Co}_{y} \mathrm{Mn}_{z} \mathrm{O}_{2}$ large format lithium ion battery. Applied Energy, 2015, 154: 74-91.

[39] Santhanagopalan S, White R E. Quantifying cell-to-cell variations in lithium ion batteries. International Journal of Electrochemistry, 2012, 2012.

[40] Dubarry M, Vuillaume N, Liaw B Y. Origins and accommodation of cell variations in Li-ion battery pack modeling. International Journal of Energy Research, 2010, 34(2): 216-231.

[41] Zheng Y, Ouyang M, Lu L, et al. Cell state-of-charge inconsistency estimation for $\mathrm{LiFePO}_{4}$ battery pack in hybrid electric vehicles using mean-difference model. Applied Energy, 2013, 111: 571-580.

[42] Kenney B, Darcovich K, MacNeil D D, et al. Modelling the impact of variations in electrode manufacturing on lithium-ion battery modules. Journal of Power Sources, 2012, 213: 391-401.

[43] Ouyang M, Gao S, Lu L, et al. Determination of the battery pack capacity considering the estimation error using a Capacity-Quantity diagram. Applied Energy, 2016, under review.

[44] Yurkovich B J, Guezennec Y. Lithium ion dynamic battery pack model and simulation for automotive applications. ASME 2009 Dynamic Systems and Control Conference. American Society of Mechanical Engineers, 2009: 241-248.

[45] Dubarry M, Vuillaume N, Liaw B Y. From single cell model to battery pack simulation for Li-ion batteries. Journal of Power Sources, 2009, 186(2): 500-507.

[46] Feng X, Weng C, Ouyang M, et al. Online internal short circuit detection for a large format lithium ion battery. Applied Energy, 2016, 177: 384-392.

[47] Christensen J, Newman J. Cyclable lithium and capacity loss in Li-ion cells. Journal of the Electrochemical Society, 2005, 152(4): A818-A829.

[48] Prada E, Di Domenico D, Creff Y, et al. A simplified electrochemical and thermal aging model of $\mathrm{LiFePO}_{4}$-graphite li-ion batteries: Power and capacity fade simulations. Journal of The Electrochemical Society, 2013, 160(4): A616-A628.

[49] Albertus P, Couts J, Srinivasan V, et al. II. A combined model for determining capacity usage and battery size for hybrid and plug-in hybrid electric vehicles. Journal of Power Sources, 2008, 183(2): 771-782.

[50] Liu P, Wang J, Hicks-Garner J, et al. Aging mechanisms of $\mathrm{LiFePO}_{4}$ batteries deduced by electrochemical and structural analyses. Journal of the Electrochemical Society, 2010, 157(4): A499-A507.

[51] Striebel K, Shim J, Sierra A, et al. The development of low cost $\mathrm{LiFePO}_{4}$-based high power lithium-ion batteries. Journal of Power Sources, 2005, 146(1): 33-38.

[52] Safari M, Delacourt C. Modeling of a commercial graphite/ $\mathrm{LiFePO}_{4}$ cell. Journal of the Electrochemical Society, 2011, 158(5): A562-A571.

[53] Shim J, Striebel K A. Cycling performance of low-cost lithium ion batteries with natural 
graphite and $\mathrm{LiFePO}_{4}$. Journal of Power Sources, 2003, 119: 955-958.

[54] Hu X, Li S, Peng H. A comparative study of equivalent circuit models for Li-ion batteries. Journal of Power Sources, 2012, 198: 359-367.

[55] Weng C, Sun J, Peng H. Model Parametrization and Adaptation Based on the Invariance of Support Vectors With Applications to Battery State-of-Health Monitoring. Vehicular Technology, IEEE Transactions on, 2015, 64(9): 3908-3917. 

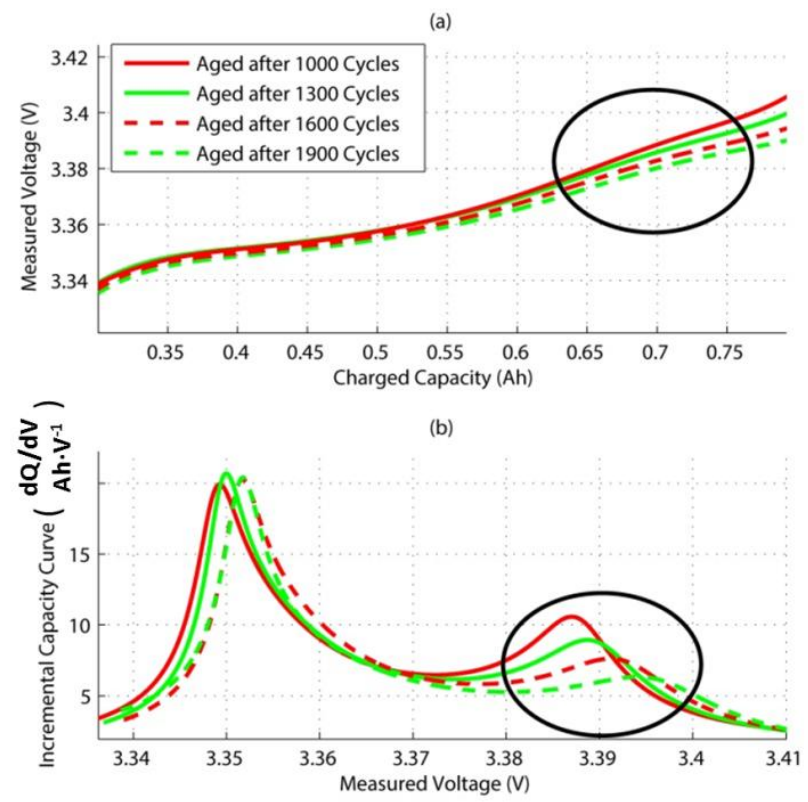

Figure 1 Aging signature extracted from $\mathrm{LiFePO}_{4}$ battery cells (charged at 1/25C) using ICA.
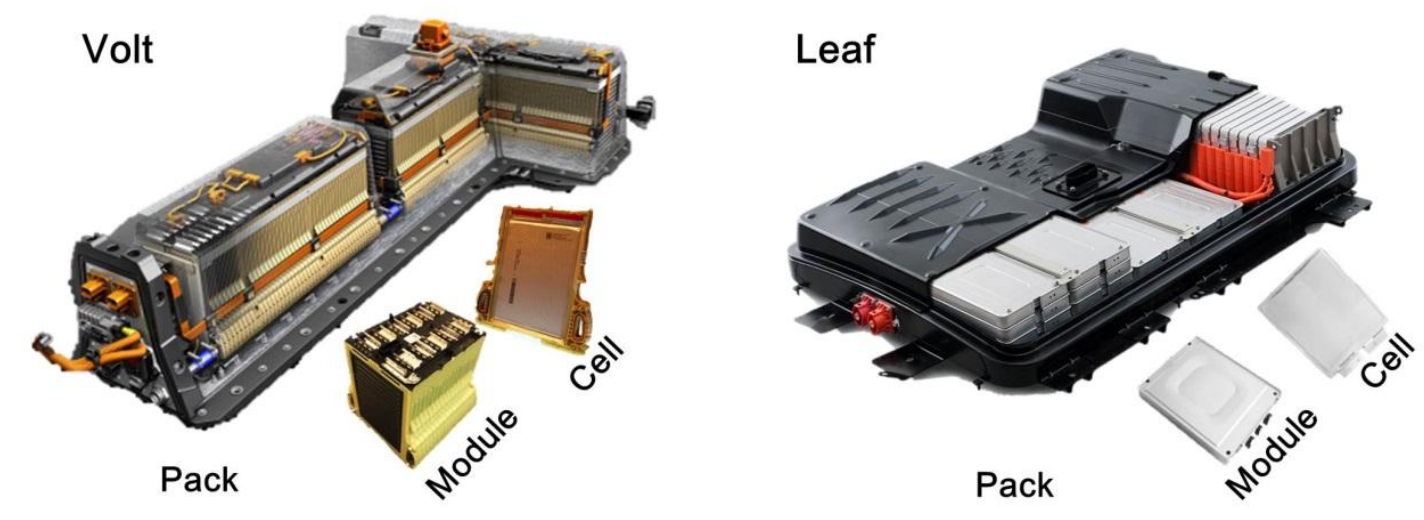

Figure 2 Battery packs of Volt and Leaf.

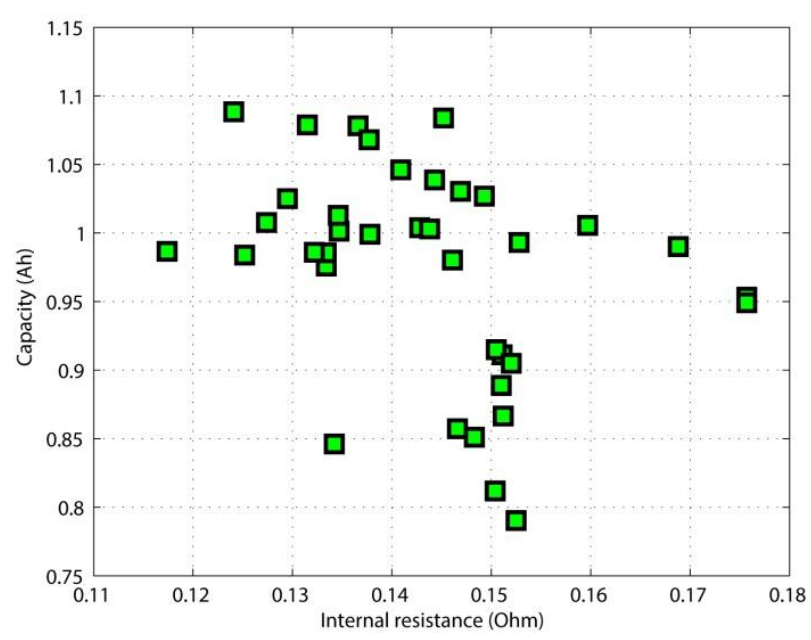

Figure 3 Capacity and resistance distribution of the test cells. 


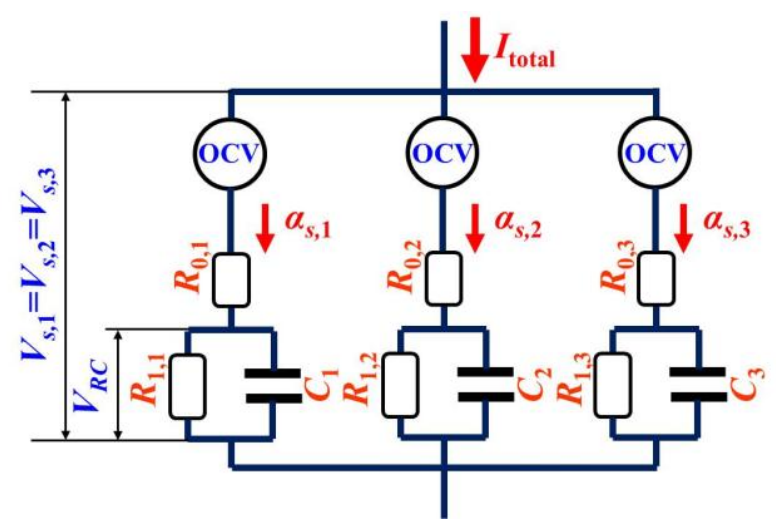

Figure 4 Battery module model used for simulations.

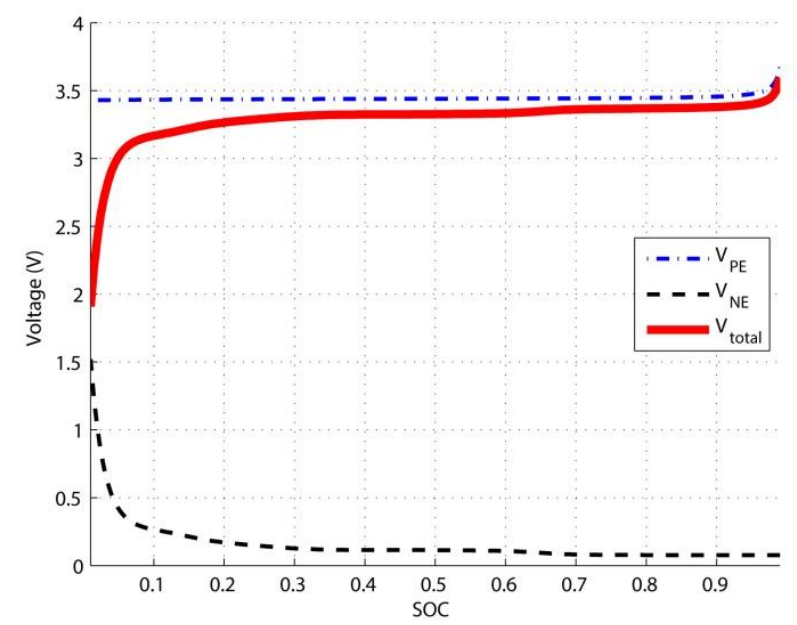

Figure 5 Open-circuit-voltage of $\mathrm{LiFePO}_{4}$ batteries simulated using the mechanistic battery aging model from Ref. [47].

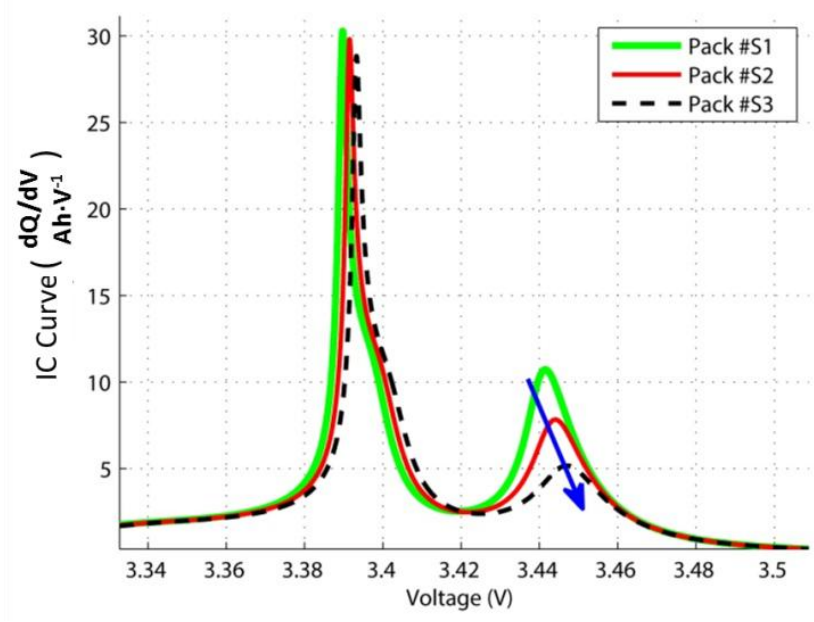

Figure 6 ICA results of battery modules simulated (\#S1, \#S2, \#S3) with variation in cell and total capacity. 


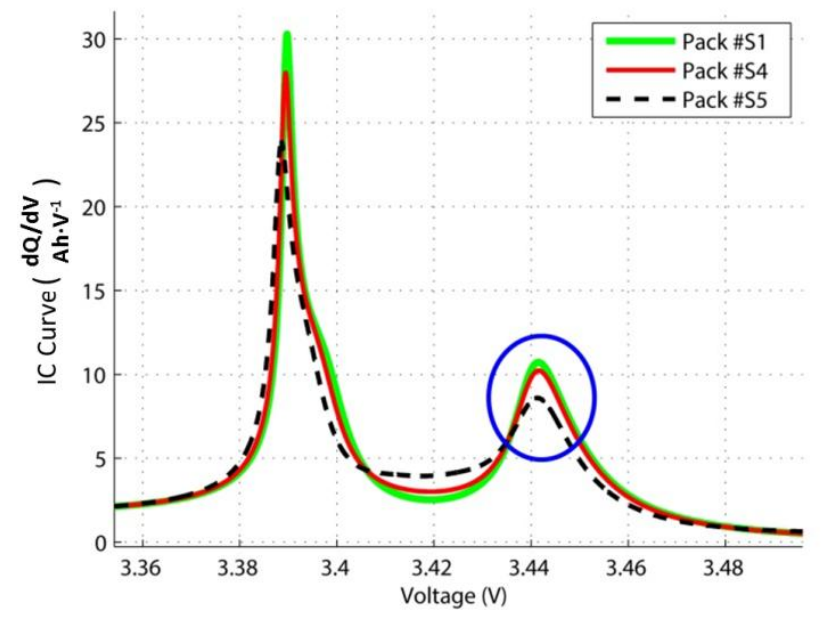

Figure 7 ICA results of battery modules (\#S1, \#S4, \#S5) simulated with variation in cell resistance.

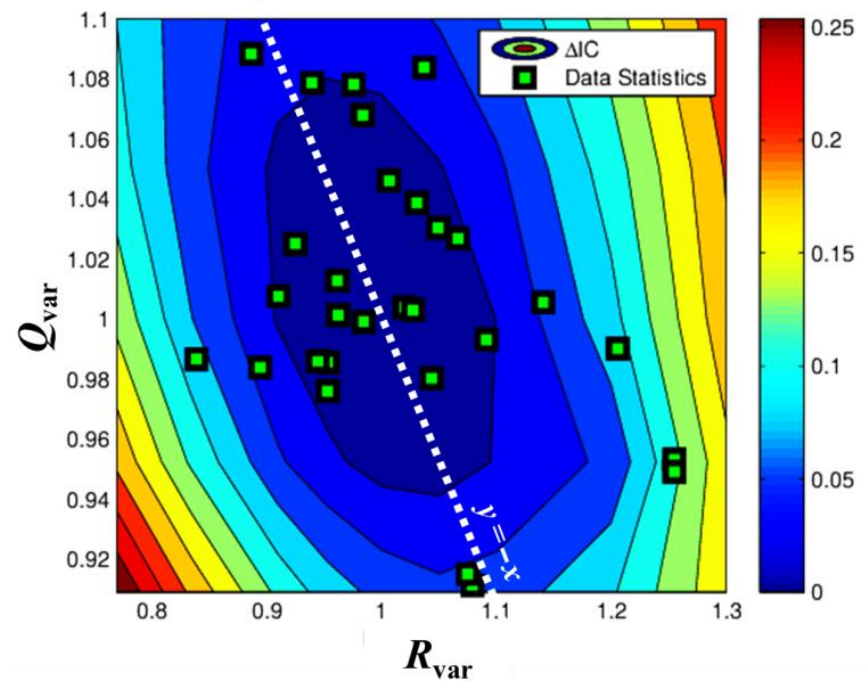

Figure 8 ICA results simulated using a 2-cell battery module with capacity and resistance variation. 


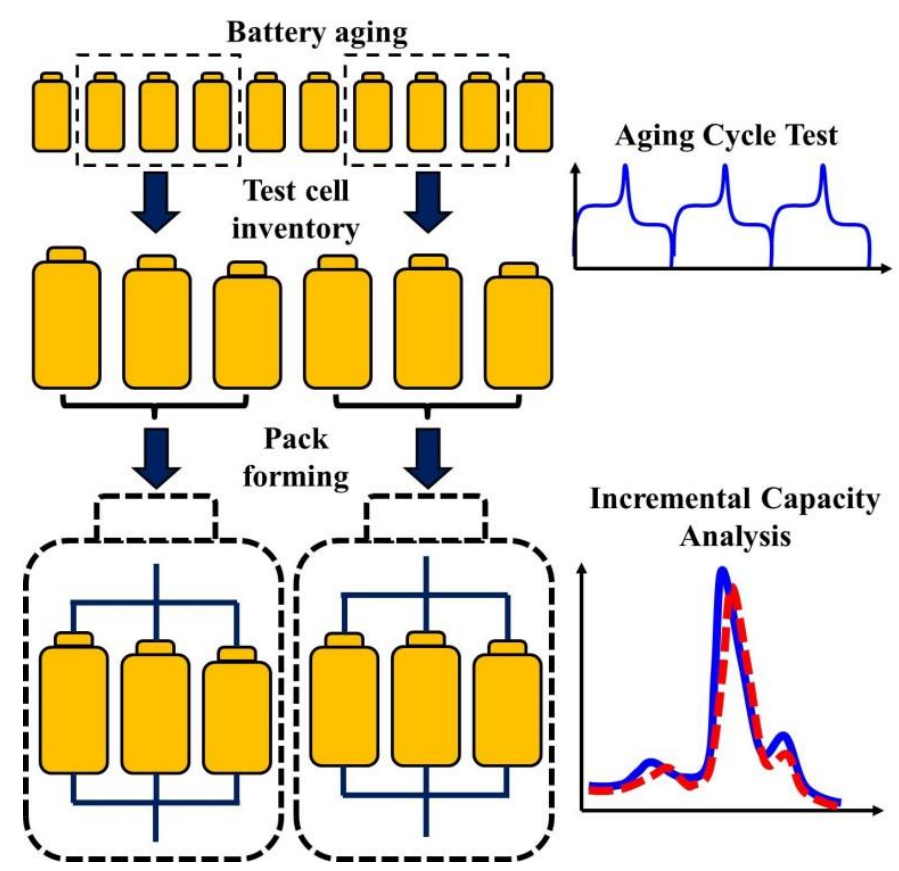

Figure 9 Design of the experimental battery module test.

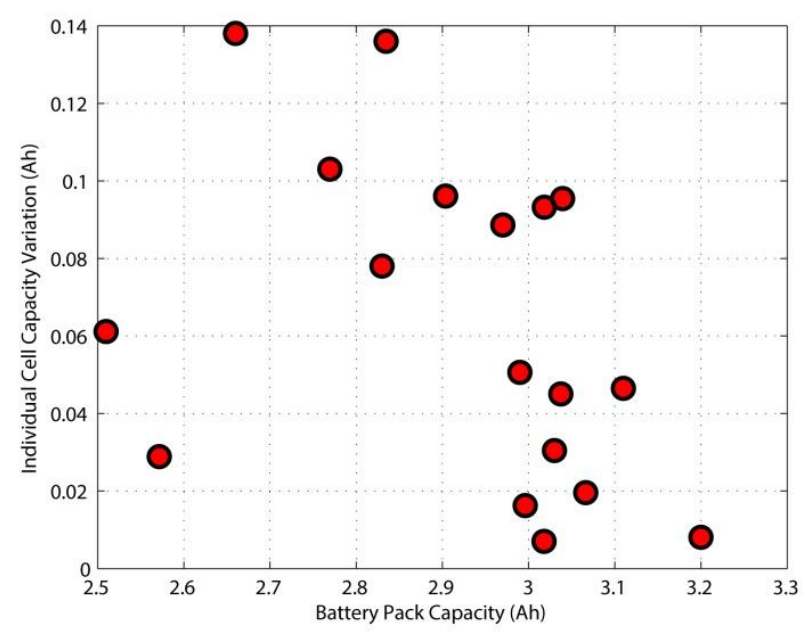

Figure 10 Characteristics of tested battery modules with 3 cells. 

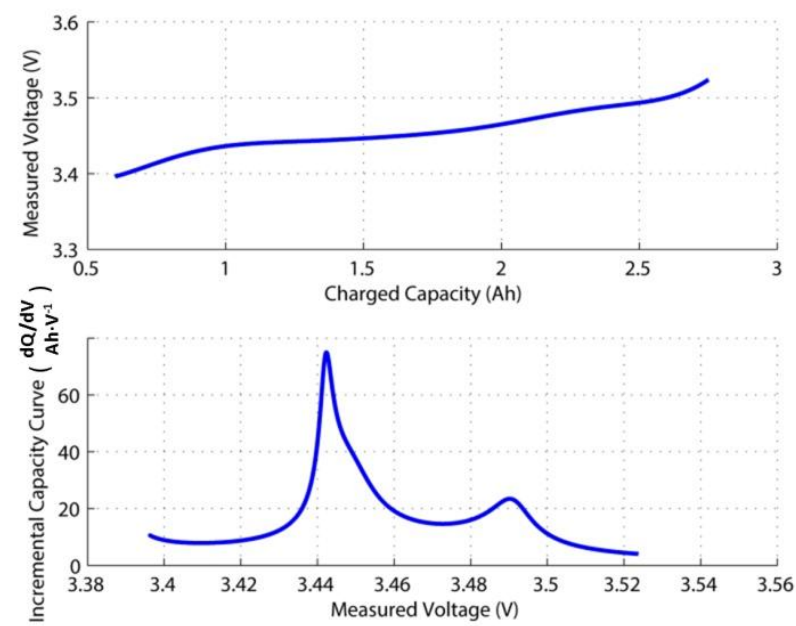

Figure 11 Computing IC curves from battery charging data using SVR algorithm.

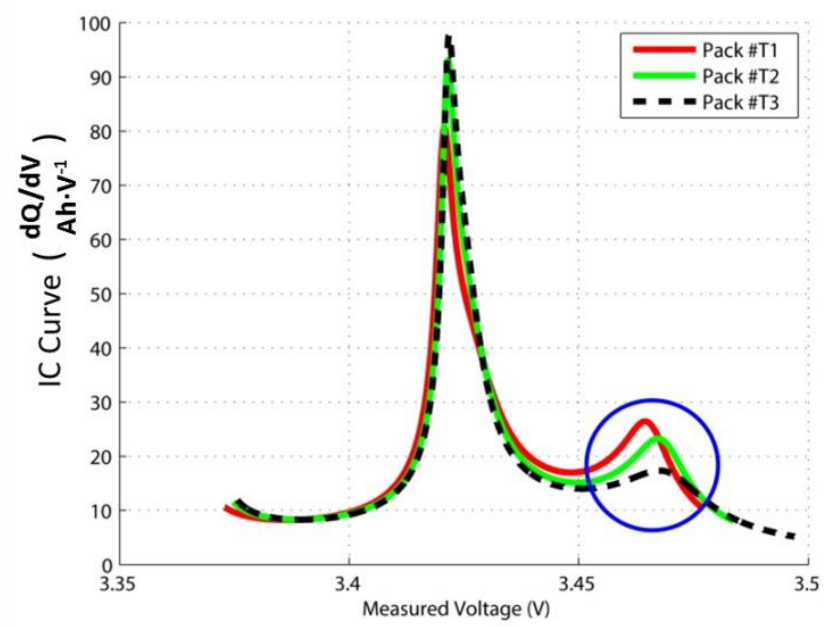

Figure 12 ICA results of 3-cell battery modules.

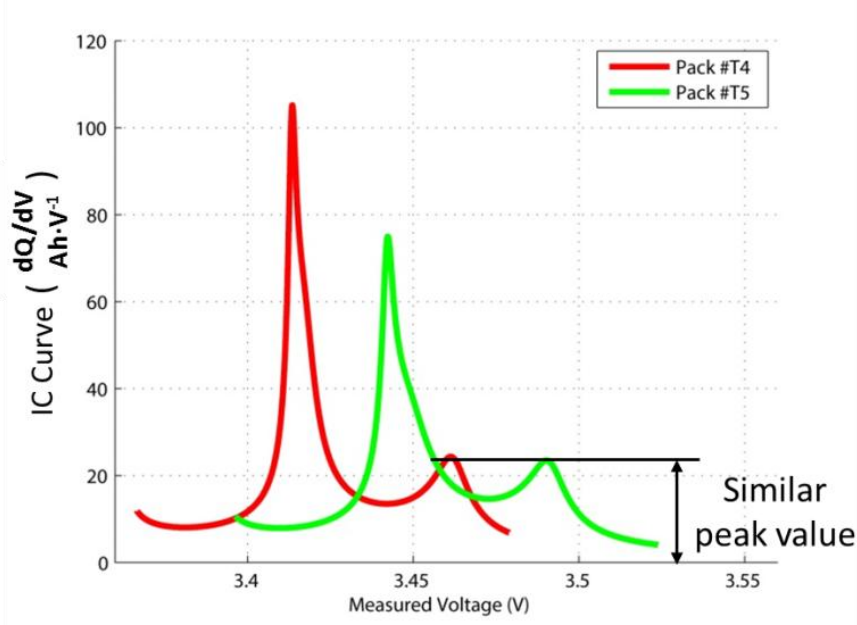

Figure 13 IC curves of battery modules with same total capacity but different cell capacities. 


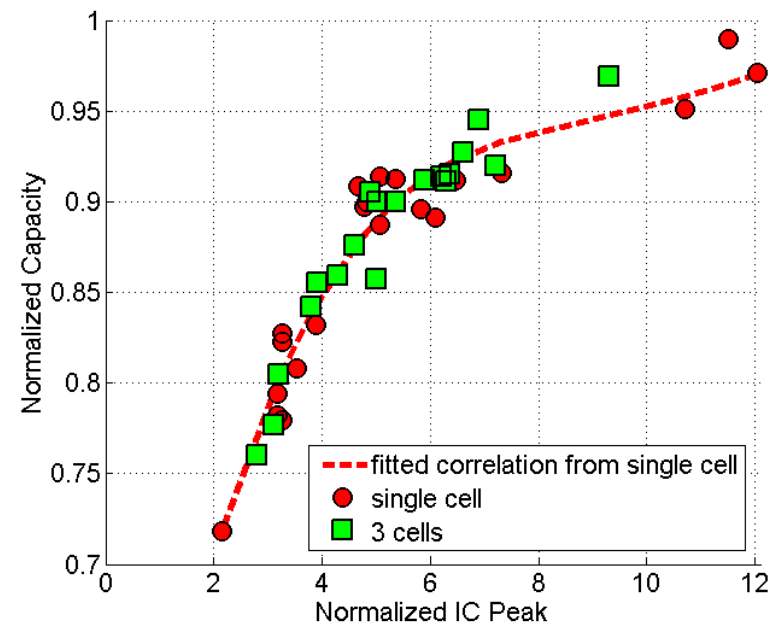

Figure 14 IC peak tracking based battery capacity estimation. 
Table 1 Main specifications of the $\mathrm{LiFePO}_{4}$ cells

\begin{tabular}{cccc}
\hline $\begin{array}{c}\text { Nominal } \\
\text { capacity }(\mathrm{Ah})\end{array}$ & $\begin{array}{c}\text { Nominal } \\
\text { voltage }(\mathbf{V})\end{array}$ & $\begin{array}{c}\text { Upper cut-off } \\
\text { voltage }(\mathbf{V})\end{array}$ & $\begin{array}{c}\text { Lower cut-off } \\
\text { voltage }(\mathrm{V})\end{array}$ \\
\hline 1.10 & 3.30 & 3.60 & 2.00 \\
\hline
\end{tabular}

Table 2 Cell parameters in battery module simulations

\begin{tabular}{ccccc}
\hline $\begin{array}{c}\text { Battery } \\
\text { module }\end{array}$ & $\begin{array}{c}\text { Total capacity } \\
(\mathbf{A h})\end{array}$ & \multicolumn{3}{c}{$\begin{array}{c}\text { Individual cell } \\
\text { Capacity }(\mathbf{A h}), \text { Resistance }(\boldsymbol{\Omega})\end{array}$} \\
\hline \#S1 & 2.85 & $0.97,0.13$ & $0.95,0.13$ & $0.93,0.13$ \\
\#S2 & 2.70 & $0.92,0.13$ & $0.90,0.13$ & $0.88,0.13$ \\
\#S3 & 2.55 & $0.87,0.13$ & $0.85,0.13$ & $0.83,0.13$ \\
\#S4 & 2.85 & $0.97,0.12$ & $0.95,0.13$ & $0.93,0.14$ \\
\#S5 & 2.85 & $0.97,0.11$ & $0.95,0.13$ & $0.93,0.15$ \\
\hline
\end{tabular}

* \#S represents simulation

Table 3 Cell parameters in battery module tests

\begin{tabular}{cccccc}
\hline $\begin{array}{c}\text { Battery } \\
\text { module }\end{array}$ & $\begin{array}{c}\text { Total } \\
\text { capacity } \\
\text { (Ah) }\end{array}$ & \multicolumn{2}{c}{$\begin{array}{c}\text { Individual cell } \\
\text { Capacity (Ah), Resistance }(\mathbf{\Omega})\end{array}$} & $\begin{array}{c}\text { Cell } \\
\text { Variation } \\
(\mathbf{A h})\end{array}$ \\
\hline \#T1 & 3.2005 & $1.0838,0.131$ & $1.0680,0.139$ & $1.0788,0.136$ & 0.008 \\
\#T2 & 3.1030 & $0.9866,0.131$ & $1.0782,0.137$ & $1.0461,0.141$ & 0.046 \\
\#T3 & 3.0179 & $1.0039,0.142$ & $0.9991,0.139$ & $0.9901,0.133$ & 0.007 \\
\#T4 & 3.0373 & $1.0251,0.134$ & $1.0059,0.135$ & $1.0076,0.138$ & 0.011 \\
\#T5 & 3.0396 & $1.0838,0.131$ & $1.0680,0.139$ & $0.9112,0.152$ & 0.095 \\
\hline
\end{tabular}

** \#T represents test

Table 4 Battery capacity estimation error

\begin{tabular}{ccc}
\hline Number of cells & $\mathbf{1}$ & $\mathbf{3}$ \\
\hline RMSE $(\%)$ & 1.62 & 1.28 \\
\hline
\end{tabular}

